\title{
The Importance of Recognizing Hibernomas as Possibly Not So Rare Pitfalls in FDG PET-A Histologically Verified Case and Literature Review
}

\author{
Thomas Leitha, Angelika Reiner-Concin, Ingrid Hurtl \\ Danube Hospital, Vienna, Austria \\ Email: thomas.leitha@wienkav.at, angelika.reiner@wienkav.at, ingrid.hurtl@wienkav.at
}

Received April 26, 2013; revised May 26, 2013; accepted June 4, 2013

Copyright (C 2013 Thomas Leitha et al. This is an open access article distributed under the Creative Commons Attribution License, which permits unrestricted use, distribution, and reproduction in any medium, provided the original work is properly cited.

\begin{abstract}
We present a glucose avid hibernoma hampering the re-staging of advanced breast cancer with FDG PET and summarize the results of the available literature. FDG PET, CT, MRI, ultrasound and histology were performed according to standardized protocols in our case. The literature search was performed on PubMed.gov. The literature search revealed 29 relevant publications starting with 2002. The high metabolic activity of hibernomas is a precarious pitfall in the staging of patients with a high pretest probability of malignancy and an increasing number of published cases indicate a possibly underestimated problem necessitating histological work-up in most cases. In our experience and in accordance with the literature unusually high uptake of a lesion and fat equivalent density in the CT should raise the suspicion for a hibernoma. The differential diagnosis between hibernomas and liposarcoma is often impossible without biopsy and histological work-up to prevent unnecessary treatment.
\end{abstract}

Keywords: Hibernoma; Pitfall; FDG PET; Literature Search; Review

\section{Introduction}

Hibernomas are rare benign tumors composed at least in part of brown fat cells, admixed in variable proportion with white adipose tissue. They arise from remnants of fetal brown fat, which is gradually replaced by white fat after the first postnatal weeks. The term is derived from the histological similarity to the brown fat found in hibernating animals [1]. Hibernomas most commonly occur where residual brown fat is found, but a wide range of anatomic locations have been described in the literature. Brown adipocytes contain a very large number of mitochondria and may exhibit an extraordinary metabolic activity [2] leading to a high FDG uptake in PET mimicking malignancy. Until recently [3] it has been postulated that hibernomas are rarely associated with another malignant tumor, but recent personal experience has led us to reconsider this topic.

\section{Case Report}

A now 63-year-old woman underwent ablation of an invasive lobular breast cancer (T1c N0 M0) of the left breast 13 years ago. 2 years earlier she experienced tu- mor relapse with axillary and cervical lymph node metastases, skin metastases and a single bone metastasis in the thoracic spine. Lymph node and skin metastases were resected followed by chemotherapy and radiotherapy (axilla and neck: $49 \mathrm{~Gy}$, thoracic spine: $8 \mathrm{~Gy}$ ). The patient was now referred to F-18 FDG PET for re-staging because of a mildly elevated tumor-marker (CA 15-3) but a negative bone scan.

Ge-68 attenuation corrected whole body 3D PET was performed with a dedicated full-ring PET scanner (ECAT EXACT, Siemens) 60 minutes after administration of 254 MBq F-18 FDG. Blood glucose level was 95 mg/dl.

The PET scan revealed a single focal uptake in the right proximal thigh with a max SUV of 10.3 (Figure 1). In the light of the previous clinical history this was regarded as highly suspicious for a soft tissue metastasis of breast cancer. External magnetic resonance tomography confirmed the potentially malignant lesion and ultrasound guided biopsy of the lesion was performed (Figure 2). In anticipation of local radiotherapy biopsy was immediately followed by CT without contrast enhancement for radiation treatment planning (Figure 3). Histology excluded malignancy and confirmed a hibernoma 


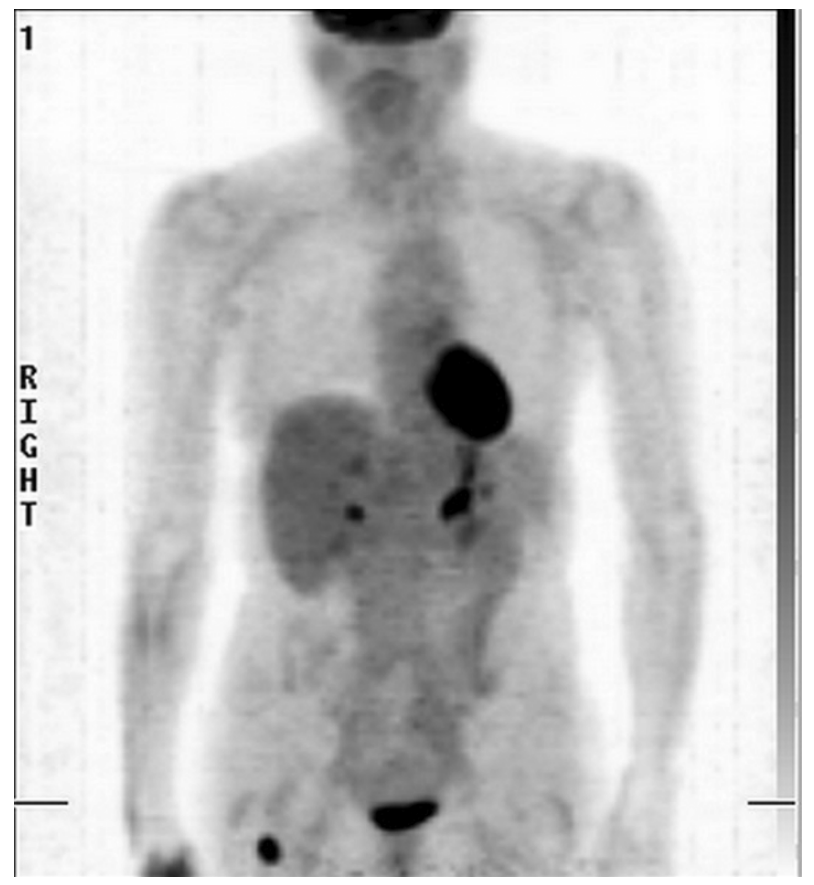

Figure 1. MIPS of FDG PET. FDG PET was negative with the exception of a hot spot (max SUV 10.4) ventrally in the right upper thigh.

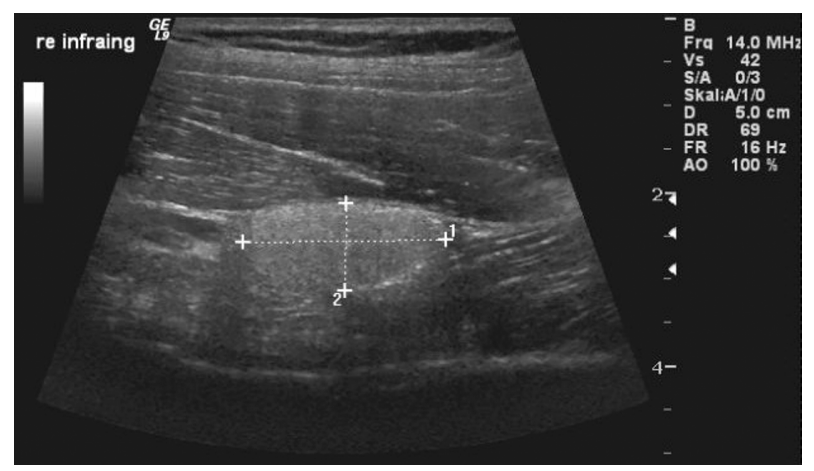

Figure 2. Ultrasound of thigh. Ultrasound showed a homogeneous, hyperechogenic soft tissue lesion and a size of $2 \times$ $1.5 \times 1.5 \mathrm{~cm}$ with sharp margins against the surrounding muscles.

(Figure 4). The tumor marker remained elevated but without significant changes during the following 8 months. No cancer treatment was administered and the patient is still regarded as being in remission.

\section{Literature Review}

A literature search was performed on the 26th April 2013 on PubMed.gov (http://www.ncbi.nlm.nih.gov/sites/entrez) with the following search term:

("lipoma”[MeSH Terms] OR “lipoma”[All Fields] OR "hibernoma”[All Fields]) AND ("positron-emission tomography”[MeSH Terms] OR (“positron-emission”[All Fields] AND “tomography”[All Fields]) OR “positron-

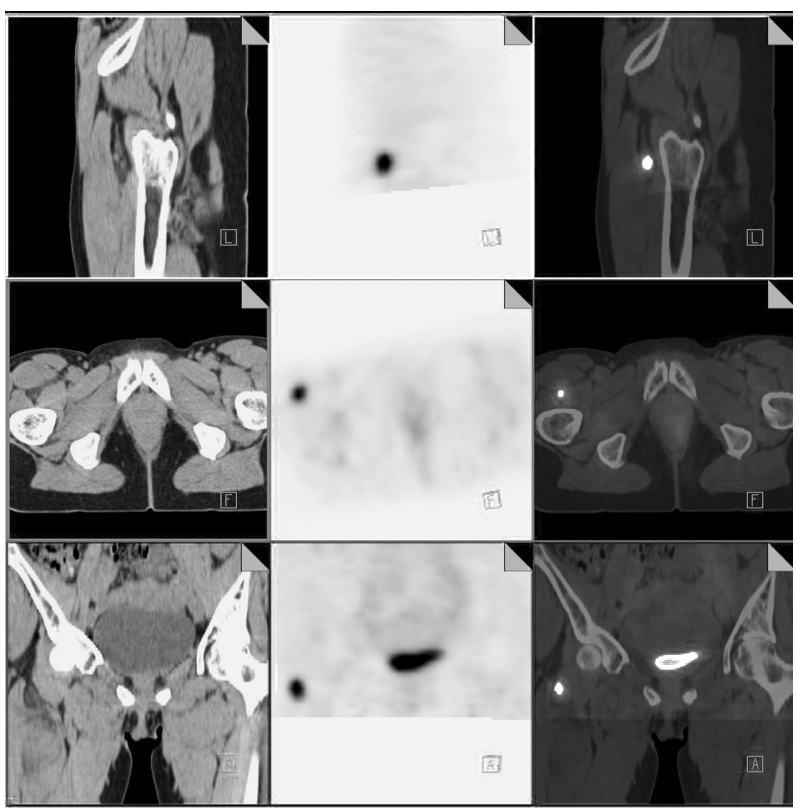

Figure 3. PET fused with non-contrast enhanced CT. Digitally fused PET and CT showed the FDG uptake in projection of a well defined hypodense lesion between vastus intermedius und medialis of the femoral muscle.

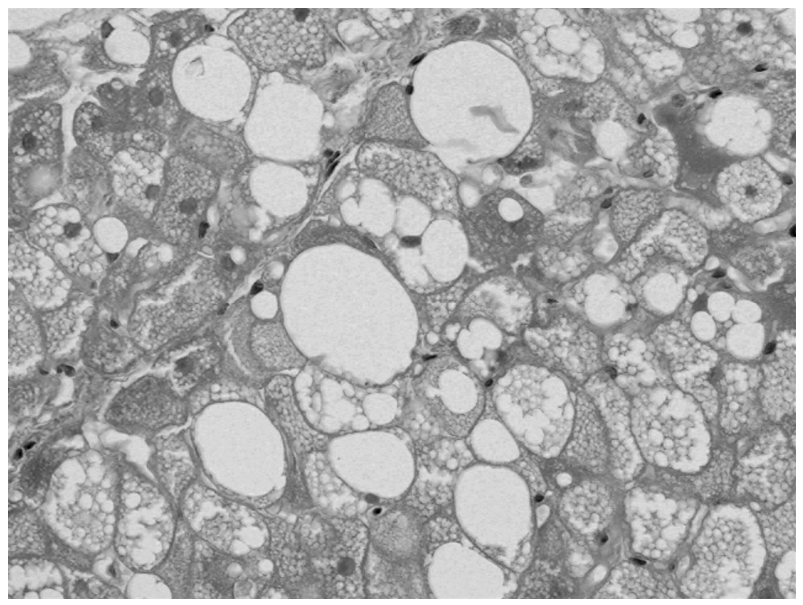

Figure 4. Histology of the lesion. The image shows large polygonal fat cells with round isomorph nuclei without mitosis. Immune staining was positive for S100 (not shown here).

emission tomography”[All Fields] OR ("fdg”[All Fields] AND “pet”[All Fields]) OR “fdg pet”[All Fields]). The results of a broader search on hibernoma (search term: "lipoma”[MeSH Terms] OR “lipoma”[All Fields] OR "hibernoma”[All Fields]); 11993 findings) were additionally used to screen for information on the natural history of hibernomas relevant for nuclear physicians.

The search revealed 38 publications on this topic, 9 studies were excluded as irrelevant. The results are summarized in Table 1. Little more than 200 cases of hibernomas have yet been published in the general lit- 
Table 1. Results of literature search.

Hibernoma grow slowly, are painless and often present as a large mass (usually 5 to $10 \mathrm{~cm}$, range: $1-33 \mathrm{~cm}$ ).

Hibernoma in many cases show a very high uptake (max SUV 7.3 26.7) in FDG PET and are nearly always misinterpreted as malignancy.

The site of uptake is no aid in differential diagnosis as beside the most common anatomic locations (neck, muscles, mediastinum, retroperitoneum... [4]) hibernomas may occur virtually everywhere. Unusual sites as the heart [5-7] and the breast [8] have been reported. Differential diagnosis to liposarcoma is difficult though these normally show low to intermediate metabolic activity in contrast to the high FDG uptake of hibernoma, but a considerable overlap exists [9-11].

It is yet unclear if two point FDG PET may aid differential diagnosis: Smith et al. [12] postulates that fluctuating SUV in follow-up scans may be diagnostic for hibernoma.

Burdick et al. [13] reported a significant rise of SUV within the lesion over time in dual time point FDG-PET.

CT and MRI are of limited utility in differential diagnosis of individual cases $[14,15]$.

erature and among those only 22 FDG positive cases were described. Noteworthy, the first report on a FDG PET positive hibernomas has been published not earlier than 2002 and since than an increasing number of cases appeared in the literature.

\section{Discussion}

The most important differential diagnosis of hibernomas presenting initially as fat containing soft tissue tumors in patients without known malignancy is liposarcoma. In contrast, nuclear physicians are more and more confronted with hibernomas in patients sent for staging of other suspected or confirmed malignancies. Since hibernomas derive from brown fat, which promotes non-shivering thermogenesis in newborns and hibernating animals, it may show a high glucose metabolism. Not surprisingly this diagnostic problem did not surface until FDG PET found widespread use in clinical oncology [3]. In the setting of patients with a high pretest probability of metastases, as in the case we presented above, chances are high of misinterpreting the high FDG uptake as evidence for metastasis and unnecessarily administer cancer therapy. The literature showed that the localization of the FDG uptake assists little in differential diagnosis. Some hibernomas occur in areas where habitually no brown fat is seen in adults, most likely due to aberrant differentiation of mesenchymal cells or by ectopic growth or migration of adipose tissue. PET-CT and MRI have limited potential in differentiating lipoma from liposarcoma. Currently it is unclear if the reported changes in FDG uptake in follow-up scans may aid in the differential diagnosis of hibernomas and metastases but the time loss seems unacceptable in cancer patients.

In our experience and in accordance with the published literature an unusually high FDG uptake (max
SUV) in circumscribed areas unrelated to the primary tumor should raise suspicion of this differential diagnosis. Thus, awareness of hibernomas as a potential pitfall in FDG PET and biopsy of dubious findings can prevent unnecessary treatment. In the light of increasing publications on this topic during the last eight years the increasing use of FDG PET apparently reveals a problem that may have been underestimated in the past.

\section{REFERENCES}

[1] I. Dardick, "Hibernoma: A Possible Model of Brown Fat Histogenesis,” Human Pathology, Vol. 9, No. 3, 1978, pp. 321-319. doi:10.1016/S0046-8177(78)80089-6

[2] H. Sell, Y. Deshaies and D. Richard, "The Brown Adipocyte: Update on Its Metabolic Role,” The International Journal of Biochemistry \& Cell Biology, Vol. 36, No. 11, 2004, pp. 2098-2104. doi:10.1016/j.biocel.2004.04.003

[3] S. Robison, A. Rapmund, C. Hemmings, M. Fulham and P. Barry, "False-Positive Diagnosis of Metastasis on Positron Emission Tomography-Computed Tomography Imaging Due to Hibernoma,” Journal of Clinical Oncology, Vol. 27, No. 6, 2009, pp. 994-995. doi:10.1200/JCO.2008.19.4977

[4] M. A. Furlong, J. C. Fanburg-Smith and M. Miettinen, "The Morphologic Spectrum of Hibernoma: A Clinicopathologic Study of 170 Cases," The American Journal of Surgical Pathology, Vol. 25, No. 6, 2001, pp. 809-814. doi:10.1097/00000478-200106000-00014

[5] P. S. Gerard, H. Finestone, R. Lazzaro and M. D. Geller, "Intermittent FDG Uptake in Lipomatous Hypertrophy of the Interatrial Septum on Serial PET/CT Scans," Clinical Nuclear Medicine, Vol. 33, No. 9, 2008, pp. 602-605. doi:10.1097/RLU.0b013e318181312a

[6] Y. Zafar and A. R. Jazieh, "Lipomatous Hypertrophy of the Interatrial Septum Evidenced on Positron Emission Tomography in a Patient with Thyroid Cancer," American Journal of Clinical Oncology, Vol. 29, No. 4, 2006, pp. 418-419. doi:10.1097/01.coc.0000170585.91248.b8

[7] C. M. Fan, A. J. Fischman, B. H. Kwek, S. Abbara and S. L. Aquino, "Lipomatous Hypertrophy of the Interatrial Septum: Increased Uptake on FDG PET," American Journal of Roentgenology, Vol. 184, No. 1, 2005, pp. 339-342. doi:10.2214/ajr.184.1.01840339

[8] N. Martini, et al. “An Unusual Breast Lesion: The Ultrasonographic, Mammographic, MRI and Nuclear Medicine Findings of Mammary Hibernoma," British Journal of Radiology, Vol. 83, No. 985, 2010, pp. 1-4. doi:10.1259/bjr/17929543

[9] J. Nishida, et al. "Clinical Findings of Hibernoma of the Buttock and Thigh: Rare Involvements and Extremely High Uptake of FDG-PET,” Medical Science Monitor, Vol. 15, No. 7, 2009, pp. CS117-CS122.

[10] T. Tsuchiya, T. Osanai, A. Ishikawa, N. Kato, Y. Watanabe and T. Ogino, "Hibernomas Show Intense Accumulation of FDG Positron Emission Tomography,” Journal of Computer Assisted Tomography, Vol. 30, No. 2, 2006, pp. 333-336. doi:10.1097/00004728-200603000-00033 
[11] R. Suzuki, et al. "PET Evaluation of Fatty Tumors in the Extremity: Possibility of Using the Standardized Uptake Value (SUV) to Differentiate Benign Tumors from Liposarcoma,” Annals of Nuclear Medicine, Vol. 19, No. 8, 2005, pp. 661-670. doi:10.1007/BF02985114

[12] C. S. Smith, J. Teruya-Feldstein, J. F. Caravelli and H. W. Yeung, "False-Positive Findings on 18F-FDG PET/CT: Differentiation of Hibernoma and Malignant Fatty Tumor on the Basis of Fluctuating Standardized Uptake Values," American Journal of Roentgenology, Vol. 190, No. 4, 2008, pp. 1091-1096. doi:10.2214/AJR.07.3061

[13] M. J. Burdick, P. R. Jolles, M. M. Grimes and D. A. Henry, "Mediastinal Hibernoma Simulates a Malignant
Lesion on Dual Time Point FDG Imaging,” Lung Cancer, Vol. 59, No. 3, 2008, pp. 391-394.

doi:10.1016/j.lungcan.2007.08.020

[14] L. W. Bancroft, M. J. Kransdorf, J. J. Peterson and M. I. O’Connor, "Benign Fatty Tumors: Classification, Clinical Course, Imaging Appearance, and Treatment,” Skeletal Radiology, Vol. 35, No. 10, 2006, pp. 719-733. doi:10.1007/s00256-006-0189-y

[15] C. M. Park, J. M. Goo, H. J. Lee, M. A. Kim, C. H. Lee and M. J. Kang, "Tumors in the Tracheobronchial Tree: CT and FDG PET Features,” RadioGraphics, Vol. 29, No. 1, 2009, pp. 55-71. doi:10.1148/rg.291085126 